\title{
Aproximación a las visiones de demostración de algunos profesores universitarios de matemáticas.
}

Artículo recibido: 31-12-2009 | Artículo aprobado:12-03-2010

An Approximation to the Conceptions Some University

Mathematics Teachers have about demonstration.

\section{Carmen Samper de Caicedo*}

Yuri Tatiana Ospina Usaquén**

Tania Julieth Plazas Merchán***
Resumen: La demostración desempeña un papel muy importante en la formación matemática de un futuro docente de esta área. Es de interés identificar las visiones que los docentes de matemáticas, de la Universidad Pedagógica Nacional, tienen respecto a dicha actividad matemática, puesto que las diferentes funciones identificadas a partir de la demostración deben presentárselas a sus alumnos, futuros profesores de matemáticas, para que desarrollen un mejor concepto de ésta. En este artículo identificamos diferencias y similitudes en esas visiones, centrándonos en las funciones que el profesor asigna a la demostración.

\begin{abstract}
Demonstration plays an important role in a pre-service teacher's mathematical training. It is interesting to identify the conceptions that Universidad Pedagógica Nacional mathematics teachers have about this teaching activity, since they should show to their students, i.e. pre-service mathematics teachers, the different functions assigned to a demonstration, so they can develop a better concept of this one. In this paper the authors identify differences and similarities among their conceptions, focusing on different functions those teachers give to a demonstration.
\end{abstract}

Palabras clave: demostración, actividad demostrativa, funciones de la demostración, enseñanza y aprendizaje de la demostración, visiones.
Keywords: Demonstration, Proving Activity, Demonstration Functions, demonstration Teachingand-Learning, Conceptions.

* Profesora Titular Universidad Pedagógica Nacional, csamper@pedagogica.edu.co

* Lic. Matemáticas Universidad Pedagógica Nacional, tatiana.ospina.usaquen@gmail.com

*** Lic. Matemáticas Universidad Pedagógica Nacional, tania.plazas.merchan@gmail.com 


\section{Introducción}

Este documento tiene como propósito presentar un reporte del trabajo de grado desarrollado para optar por el título de Licenciatura en Matemáticas de la Universidad Pedagógica Nacional. El objetivo de éste era caracterizar las visiones sobre la actividad matemática de demostrar que tienen algunos docentes del Departamento de Matemáticas de la Universidad Pedagógica Nacional, y cómo estas visiones se involucran en su práctica. El estudio fue realizado durante dos semestres.

Por medio de una entrevista de ocho (8) preguntas realizada a once profesores, se indagó acerca del manejo, importancia y función que ellos le atribuyen a la demostración. Se realizó un análisis descriptivo de las respuestas que cada docente dio a las preguntas de la entrevista con el fin de encontrar características comunes en las ideas de cada uno de ellos. Además, se hizo un registro audiovisual de una clase, de algunos profesores, en la cual se realizaron demostraciones $y$, a partir de ello, un análisis cualitativo de éstos con la intención de realizar una comparación entre sus ideas teóricas y su práctica.

\section{Marco teórico}

Thompson (1992) considera como concepción aquellas opiniones conscientes o subconscientes, significados, reglas y preferencias que tiene el profesor respecto a algo. En el caso de la educación matemática, concepciones son aquellas consideraciones que hace el docente sobre su papel en el aula, el papel de sus estudiantes, el aprendizaje que se puede propiciar a través de las actividades que lleva al aula, lo que considera pertinente en su instrucción, y su punto de vista de cómo aprenden los estudiantes. En este documento hablaremos de la visión de los profesores respecto a la demostración, ya que las herramientas de recolección de datos no son lo suficientemente amplias para poder establecer la correspondiente concepción; es decir, sólo podemos hacer un acercamiento a lo que es dicha concepción.

Vamos a tener en cuenta tres visiones propuestas por Godino y Recio (2001), y Recio (2001) para la demostración que son: desde la lógica bivalente, desde los demás campos de la matemática y desde la educación matemática. Respecto a la primera, denominada también la visión clásica, ellos resaltan que en ella la demostración está sujeta a un sistema axiomático y al pensamiento formal deductivo. La demostración juega un papel importante en la construcción de un sistema axiomático, pues a partir de unas premisas, axiomas, postulados, teoremas y definiciones usando las reglas de la lógica formal bivalente se deducen otros hechos que se incorporan a dicho sistema. Los teoremas que ingresan al sistema axiomático son considerados válidos en la teoría matemática correspondiente, sin importar el contexto de las proposiciones que componen el teorema, ya que están ligados a la validez de las reglas de la lógica. Por ejemplo, $\left[\left(p^{\circledR} q\right) \text { Ù } p\right]^{\circledR} q$ es un teorema de toda teoría matemática que se basa en la lógica bivalente usual. La demostración en este contexto se reduce a una actividad algorítmica que no involucra un contexto específico.

Según Godino y Recio (2001), la demostración vista desde los demás campos de la matemática como, por ejemplo, el álgebra o el análisis matemático aplicado a otras ciencias, como la estadística, está ligado a un sistema axiomático con un contexto teórico definido y al pensamiento formal deductivo. Esta visión sitúa la demostración en un ámbito donde el centro no es el análisis 
Aproximación a las visiones de demostración de algunos profesores universitarios de matemáticas Carmen Samper de Caicedo | Yuri Tatiana Ospina Usaquén | Tania Julieth Plazas Merchán

sintáctico de las proposiciones, es decir, su composición lógica, sino que se enfoca principalmente en las interpretaciones matemáticas que asumen las proposiciones dentro de una teoría. Las funciones de la demostración son, de cierta forma, subjetivas porque dependen de la visión que tenga la persona acerca de ella. Los teoremas dependen del sistema axiomático y de la aceptabilidad de la demostración dentro de una comunidad específica.

Por último, hablar de la demostración desde la educación matemática implica la generación de discusiones sobre el papel de ésta en la formación matemática de un individuo, siendo éste el aspecto que ofrece algunos referentes teóricos para el posterior análisis de las visiones de los profesores acerca de la demostración. Según Recio (2001), éstas se dividen en dos aspectos: unas ligadas a cómo el docente hace uso de la demostración en sus clases, la demostración en la enseñanza, y las otras relacionadas a cómo se deben generar ambientes para que los estudiantes aprendan a demostrar, la enseñanza de la demostración. En el primer aspecto se analiza el propósito de una demostración en la labor del docente en el aula de clase; es decir cómo se hace uso de ella para lograr un propósito específico. Recio sugiere dos situaciones: (1) "la demostración como parte esencial del contrato didáctico en [la clase de] matemáticas" y (2) la demostración como "una fuente insustituible de entrenamiento". La primera se refiere a la no aceptación, por parte de los estudiantes, de proposición alguna sin su demostración inmediata, haciendo que las proposiciones no demostradas pasen a un segundo nivel, o que se conviertan en parte de la segunda situación. La segunda se refiere a la importancia que le asigna el profesor a que los estudiantes conozcan las demostraciones de las proposiciones que se enuncian, pues considera que ellos deben analizar y comprender las mismas. De cierta manera, piensan que si no se demuestra no se aprende a demostrar.

En cuanto al segundo aspecto, la enseñanza de la demostración, ello es uno de los temas de interés de educadores matemáticos. Existen diferentes ideas en torno a cómo debe ser el proceso de enseñanza. Generalmente, los profesores proveen a sus estudiantes una visión limitada de qué es demostrar, pues esa actividad en sus clases se reduce a realizar ejemplos de demostraciones que los estudiantes deben entender y replicar (Recio 2001). De Villiers (1993) expresa la necesidad de transformar el paradigma que tienen muchos profesores respecto a la demostración:

La idea común formalista de muchos profesores de matemáticas de que la convicción es una cartografía monocromática de la demostración deductiva (esto es: una función biyectiva) debe ser, por tanto, completamente abandonada; la convicción no se consigue exclusivamente con la demostración ni es la verificación/convicción la única función de la demostración. (de Villiers, 1993)

Pensar en las funciones de la demostración es reflexionar sobre los aportes de la misma en la formación de un individuo. En el presente tendremos en cuenta los aportes que de Villiers (1993) ofrece en relación con las funciones de la demostración. Este autor destaca cinco funciones principales: verificación/convicción, explicación, sistematización, descubrimiento y comunicación. Él cree que la función que hace significativa la demostración en el aula de clase es la explicación/comprensión, la cual está estrechamente ligada a las funciones de comunicación y descubrimiento. En la siguiente tabla se describe cada una de estas funciones (Tabla 1). 


\begin{tabular}{|c|c|}
\hline Función & Descripción \\
\hline Verificación/convicción & $\begin{array}{l}\text { Tiene como objetivo obtener certeza de un teorema } \\
\text { y convencer a los pares y a uno mismo de ésta. Esta } \\
\text { función exige demostraciones formales y completas, } \\
\text { cuando se trata de un grupo de expertos. Pero, ello } \\
\text { puede modificarse según el nivel académico del grupo } \\
\text { de personas. }\end{array}$ \\
\hline Explicar/comprender & $\begin{array}{l}\text { Busca proveer el por qué la proposición es verdadera. Se } \\
\text { utiliza con el propósito de que el individuo comprenda } \\
\text { mejor el objeto matemático involucrado. Usualmente } \\
\text { esta función cobra mayor importancia cuando se } \\
\text { piensa en el papel de la demostración en la formación } \\
\text { matemática de un individuo. }\end{array}$ \\
\hline Sistematización & $\begin{array}{l}\text { Atiende a la organización de los axiomas, definiciones } \\
\text { y teoremas dentro de una teoría. Tiene las siguientes } \\
\text { intenciones: identificar inconsistencias, integrar los } \\
\text { teoremas y definiciones, dar lugar a nuevos sistemas } \\
\text { axiomáticos, permitir coherencia y unificación de los } \\
\text { resultados. }\end{array}$ \\
\hline Descubrimiento/exploración & $\begin{array}{l}\text { Tiene como objetivo encontrar nuevos teoremas a partir } \\
\text { de deducciones de otros teoremas, como ha sucedido } \\
\text { históricamente, o a partir de la exploración y análisis de } \\
\text { situaciones. }\end{array}$ \\
\hline Comunicación & $\begin{array}{l}\text { Busca informar resultados matemáticos entre personas } \\
\text { de una misma comunidad. Por lo tanto, es una actividad } \\
\text { social que estipula ciertas normas para que el proceso } \\
\text { comunicativo sea óptimo. Permite vislumbrar las demás } \\
\text { funciones de la demostración, ya que si no existiera } \\
\text { interés en comunicarla no habría interés en convencer, } \\
\text { explicar y sistematizar. }\end{array}$ \\
\hline
\end{tabular}

Tabla 1. Tipos de funciones

La visión de la demostración que ofrece el grupo de investigación Aprendizaje y Enseñanza de la Geometría ( $\mathbb{E} \cdot G$ ), de la Universidad Pedagógica Nacional en Camargo, Samper y Perry (2006), resalta que ésta última es medio de descubrimiento, comunicación, explicación y sistematización. El grupo propone lo que denominan actividad demostrativa-la cual se compone de dos procesos: (1) construcción de conjeturas y (2) producción de justificaciones. El primer proceso incluye acciones como la visualización, exploración, generalización y verificación para poder establecer conjeturas. El segundo proceso se centra en la justificación ya sea a través de una explicación, una prueba o una demostración formal. La actividad demostrativa tiene como fin des- 
Aproximación a las visiones de demostración de algunos profesores universitarios de matemáticas Carmen Samper de Caicedo | Yuri Tatiana Ospina Usaquén | Tania Julieth Plazas Merchán

cubrir hechos geométricos, propiciar mejor comprensión de la propiedad y el objeto matemático trabajado, y proveer elementos para la construcción de una justificación.

\section{Metodología}

La población de estudio se conformó de once (11) profesores del Departamento de Matemáticas de la Universidad Pedagógica Nacional que se encontraban laborando, durante los periodos académicos correspondientes al segundo semestre de 2008 y el primer semestre de 2009, como profesores de algunos de los espacios académicos de matemáticas, de la Licenciatura en Matemáticas. Se escogieron esos profesores y no aquellos a cargo de los cursos de la línea de didáctica de las matemáticas, debido a que se quería comparar las opiniones que daban sobre la demostración con su puesta en práctica en el aula.

La recolección de información se realizó en dos fases:

- Fase 1 (Entrevista): Se diseñó un instrumento tipo entrevista semiestructurada, con ocho preguntas diseñadas con el fin de obtener información sobre diferentes aspectos relacionados con la demostración (Tabla 2). A través de las respuestas, se buscaba identificar las funciones que el docente le asigna a la demostración en la matemática y en la educación matemática.

\begin{tabular}{|c|c|}
\hline Pregunta & Propósito \\
\hline $\begin{array}{l}\text { 1. ¿Qué es demostrar? } \\
\text { 2. ¿Es importante la demostración en mate- } \\
\text { máticas? ¿Por qué? }\end{array}$ & $\begin{array}{l}\text { Lograr una aproximación a lo que conside- } \\
\text { ran como demostración, desde su forma- } \\
\text { ción matemática. }\end{array}$ \\
\hline $\begin{array}{l}\text { 3. ¿Cuál es el papel de la demostración en } \\
\text { el aprendizaje de las matemáticas? }\end{array}$ & $\begin{array}{l}\text { Evidenciar si diferencian entre lo que es la } \\
\text { demostración para los matemáticos y lo } \\
\text { que es la demostración en el proceso de } \\
\text { aprender matemáticas. }\end{array}$ \\
\hline $\begin{array}{l}\text { 4. ¿Cómo cree que un estudiante aprende a } \\
\text { demostrar? } \\
\text { 5. ¿Es posible enseñar a demostrar? ¿Cómo } \\
\text { lo hace usted? }\end{array}$ & $\begin{array}{l}\text { Determinar si reconocen la problemática } \\
\text { asociada al aprendizaje de la demostración } \\
\text { y si tienen una propuesta didáctica para } \\
\text { abordarla. }\end{array}$ \\
\hline $\begin{array}{l}\text { 6. ¿Es importante la demostración en la for- } \\
\text { mación de un maestro? ¿Por qué? } \\
\text { 7. ¿Considera usted que la demostración } \\
\text { se debe llevar a nivel escolar? ¿Cómo se } \\
\text { debe implementar la demostración en } \\
\text { este nivel? }\end{array}$ & $\begin{array}{l}\text { Establecer la visión que tiene de la demos- } \\
\text { tración en la educación matemática de los } \\
\text { individuos, y el papel que juega un maestro } \\
\text { en propiciar habilidades que están ligadas a } \\
\text { la demostración, a nivel escolar. }\end{array}$ \\
\hline $\begin{array}{l}\text { 8. ¿Existe alguna diferencia entre demos- } \\
\text { trar en álgebra, demostrar en geometría } \\
\text { y demostrar en cálculo }\end{array}$ & $\begin{array}{l}\text { Evidenciar mayor amplitud de su visión } \\
\text { acerca de la demostración, dado que } \\
\text { podían asignarle diferentes funciones } \\
\text { según el campo teórico en el que se realiza } \\
\text { la demostración. }\end{array}$ \\
\hline
\end{tabular}

Tabla 2. Preguntas de la entrevista y sus propósitos 
Se realizó la entrevista a todos los profesores escogidos, durante el segundo semestre de 2008. De ésta se hizo un registro de audio, el cual posteriormente fue transcrito y analizado para establecer las tipologías en las que serían clasificados los docentes.

- Fase 2 (Observación): En esta fase se tenía como meta realizar un registro audiovisual de una clase, de cada uno de los profesores entrevistados, en la cual se realizara por lo menos una demostración. Sin embargo, por cuestiones relacionadas con sucesos en la universidad que interrumpieron la normalidad académica, sólo fue posible hacer este registro a tres de los profesores. Uno de ellos tenía a su cargo un espacio académico de cálculo, otro docente un espacio académico de geometría y el último un espacio académico de álgebra. Para el análisis de este registro se tuvieron en cuenta ocho aspectos relacionados con la gestión del profesor durante el proceso de demostración (Tabla 3):

\begin{tabular}{|l|l|}
\hline \multicolumn{1}{|c|}{ Aspectos } & \multicolumn{1}{|c|}{ Propósito } \\
\hline $\begin{array}{l}\text { Deja participar a los estudiantes en la construcción } \\
\text { de demostraciones }\end{array}$ & $\begin{array}{l}\text { Evidenciar su visión del papel de } \\
\text { la demostración en la educación } \\
\text { matemática de un individuo. }\end{array}$ \\
\hline $\begin{array}{l}\text { Indaga para propiciar participación de estudiantes } \\
\text { con ideas para la demostración }\end{array}$ & $\begin{array}{l}\text { Establecer las ideas que tiene sobre lo } \\
\text { que es aprender a demostrar y enseñar a } \\
\text { demostrar. }\end{array}$ \\
\hline $\begin{array}{l}\text { Busca la comprensión de los estudiantes } \\
\text { presentando ejemplos o contraejemplos antes de } \\
\text { demostrar el teorema }\end{array}$ & $\begin{array}{l}\text { Determinar el papel que le asigna a la } \\
\text { demostración en el aprendizaje de las } \\
\text { matemáticas. }\end{array}$ \\
\hline $\begin{array}{l}\text { Usa expresiones que se refieren a la demostración } \\
\text { como validación/convicción (¿Cómo se puede } \\
\text { validar esto?, ¿Cómo puedo mostrar que esto es } \\
\text { cierto?) } \\
\begin{array}{l}\text { Usa expresiones que se refieren a la demostración } \\
\text { para la sistematización. (Se debe primero demostrar } \\
\text { esto..., esto es cierto por el teorema anterior... } \\
\text { Usa expresiones que se refieren a la demostración } \\
\text { para la comprensión o realiza acciones a través de } \\
\text { las cuales se entrevé que considera la demostración } \\
\text { elemento para la comprensión/explicación } \\
\text { Da lugar a un espacio de actividad previa para } \\
\text { investigar una situación que lleve al descubrimiento } \\
\text { del teorema a demostrar. }\end{array}\end{array}$ & $\begin{array}{l}\text { Capturar la información que permita } \\
\text { evidenciar las funciones que le otorga a } \\
\text { la demostración en su práctica docente. }\end{array}$ \\
\hline $\begin{array}{l}\text { Problematiza el teorema a demostrar con el fin de } \\
\text { generar discusiones en torno a éste. }\end{array}$ & $\begin{array}{l}\text { Observar estrategias didácticas para } \\
\text { la enseñanza y el aprendizaje de la } \\
\text { demostración. }\end{array}$ \\
\hline $\begin{array}{l}\text { Muestra interés en la refinación y rigurosidad de la } \\
\text { demostración }\end{array}$ & $\begin{array}{l}\text { Evidenciar si le asigna a la demostración, } \\
\text { desde la Educación Matemática, un } \\
\text { papel diferente al que le corresponde en } \\
\text { la matemática. }\end{array}$ \\
\hline
\end{tabular}

Tabla 3. Criterios para el análisis del registro. 
Aproximación a las visiones de demostración de algunos profesores universitarios de matemáticas Carmen Samper de Caicedo | Yuri Tatiana Ospina Usaquén | Tania Julieth Plazas Merchán

\section{Análisis de resultados}

A partir de las respuestas de cada profesor a las preguntas de la entrevista, se consolidó una tipología de acuerdo al número de funciones de la demostración que le asignó y a su visión acerca de la enseñanza y aprendizaje de la demostración. De acuerdo a ésta, se realizó una primera clasificación de los profesores. Finalmente, teniendo en cuenta el análisis de la información recolectada en los registros audiovisuales, se modificó la clasificación inicial de los docentes.

- Fase 1 (Análisis de las respuestas a las preguntas de la entrevista) A continuación se presentan las conclusiones que se obtuvieron de las respuestas a cada pregunta.

\section{1. ¿Qué es demostrar?}

Ocho profesores consideran a la demostración como una sucesión de afirmaciones debidamente justificadas y entrelazadas, que culminan en el consecuente de la proposición a demostrar. Ello los sitúa en la visión, descrita en el marco teórico, que corresponde a la demostración desde los demás campos de la matemática. Dos de los docentes explícitamente manifiestan que la demostración es una construcción lógica, y tres de ellos definen de manera explícita la demostración a partir de algunas de sus funciones.

2. ¿Es importante la demostración en matemáticas? ¿Por qué?

En las respuestas fue posible vislumbrar las primeras ideas que tienen los profesores acerca de las funciones de la demostración, siendo las que mencionan convencer, comprender y verificar. Asignar a la demostración la función de verificar o convencer es un aspecto común de los docentes del Departamento, ya que ocho de once resaltan estas funciones. Dos de los docentes ven a la demostración como un mecanismo de convencimiento. De la respuesta del otro profesor no fue posible determinar las funciones que le asigna a la demostración.

3. ¿Cuál es el papel de la demostración en el aprendizaje de las matemáticas?

Al reflexionar sobre los aspectos relacionados con la demostración en la formación matemática de sus estudiantes, cinco profesores mencionan otras funciones de la demostración que en el momento de definirla no tuvieron en cuenta. Por ejemplo, añaden "comprender" y "aprender a argumentar" a la función tradicional de validar/ convencer. Seis docentes mencionan su uso para "formar en el rigor" $\mathrm{y}$ "aprender qué es matemáticas" considerando que la demostración es parte del contrato didáctico que establece en el aula.

4. ¿Es posible enseñar a demostrar? ¿Cómo lo hace usted?

Ocho profesores creen que la demostración se debe enseñar "presentando ejemplos de demostraciones" y "dando pautas". Esto indica que tienen una visión cerrada de la demostración, pues sólo ven en ella la función de verificación/convicción, dejando de lado las demás funciones que aportan elementos diferentes a la formación de un individuo. Los tres docentes que hacen referencia a métodos de enseñanza como "un proceso de explorar, conjeturar, justificar", resaltan más funciones de la demostración, aceptando la actividad demostrativa como un constructo que satisface cada una de las funciones que le otorgan. Además, incorporan en sus ideas la función de descubrimiento (exploración). 
5. ¿Cómo cree que un estudiante aprende a demostrar?

Los nueve docentes que mencionan como proceso de aprendizaje "haciendo demostraciones, con ejemplos de demostraciones" y "siguiendo un proceso de análisis, examinando ejemplos" siguen viendo la demostración como una actividad de entrenamiento. Dos profesores concuerdan con las ideas expresadas en la pregunta anterior, al especificar que los estudiantes aprenden a demostrar "por medio de un proceso de explorar, conjeturar, y justificar" siendo ellos quienes además manifiestan que es posible enseñar a demostrar. Esto porque ellos ven a la demostración como un proceso amplio y no como un producto solamente. Por lo tanto, cuando hablan de la enseñanza de la demostración, hacen referencia a la generación de ambientes que deben propiciar el aprendizaje de los estudiantes.

6. ¿Es importante la demostración en la formación de un maestro? ¿Por qué?

Dos profesores identifican la función de explicar/comprender en sus respuestas. Ello se evidencia cuando indican que si el estudiante para profesor comprende los objetos matemáticos, el tratamiento que le darán a éstos en sus clases será más accesible, claro y acorde con la teoría. Cinco profesores mencionan que la importancia radica en la función de convencer/verificar, y los demás en que es un método para aprender matemáticas y a argumentar.

7. ¿Considera usted que la demostración se debe llevar a nivel escolar? ¿Cómo se debe implementar la demostración en este nivel?

Nueve profesores consideran que hay diferentes niveles de rigor para la justifica- ción, y que sólo cuando se tiene un nivel conceptual y de abstracción superior al que se puede manejar en la escuela, es posible hacer lo que consideran realmente como demostración. Afirman que a nivel escolar se hacen justificaciones informales. Dos de estos docentes explícitamente dicen que se deben realizar procesos de exploración, conjeturación y justificación por medio de explicaciones que pueden ser empíricas. Los otros dos docentes creen que la posibilidad de hacer demostraciones a nivel escolar depende de la posibilidad de hacer representaciones para contar con el apoyo visual y sólo en los cursos superiores de la escuela.

8. ¿Existe alguna diferencia entre demostrar en álgebra, demostrar en geometría y demostrar en cálculo?

Todos los docentes concuerdan en que la estructura de la demostración, bajo las reglas lógicas, es la misma. Sin embargo, encuentran que una diferencia sustancial está en la complejidad del sistema axiomático y en los recursos a disposición en la teoría que se necesitan para hacer la demostración o para hacer representaciones que apoyen el proceso de demostrar. Algunos afirman que la dificultad para demostrar, se tendría, de menor a mayor, en: álgebra, geometría y cálculo.

A continuación se presenta la interpretación que se hizo de las respuestas a las preguntas, con respecto a las funciones que los profesores le asignan a la demostración, de acuerdo con lo expuesto en el marco teórico. Es de aclarar que en la entrevista no se preguntó de manera explícita sobre cuál(es) es (son) la(s) función(es) que le atribuyen a la demostración, pero en sus respuestas se entrevén las funciones que le asignan a ella (Tabla 4). 
Aproximación a las visiones de demostración de algunos profesores universitarios de matemáticas Carmen Samper de Caicedo | Yuri Tatiana Ospina Usaquén | Tania Julieth Plazas Merchán

\begin{tabular}{|l|c|c|c|c|c|c|c|c|c|c|c|}
\hline Función Profesor & A & B & C & D & E & F & G & H & I & J & K \\
\hline $\begin{array}{l}\text { Verificación/convic- } \\
\text { ción }\end{array}$ & $\mathrm{X}$ & $\mathrm{X}$ & $\mathrm{X}$ & $\mathrm{X}$ & $\mathrm{X}$ & $\mathrm{X}$ & $\mathrm{X}$ & $\mathrm{X}$ & $\mathrm{X}$ & $\mathrm{X}$ & $\mathrm{X}$ \\
\hline Sistematización & $\mathrm{X}$ & & & & & & & & $\mathrm{X}$ & & $\mathrm{X}$ \\
\hline $\begin{array}{l}\text { Descubrimiento/ } \\
\text { exploración }\end{array}$ & & & & & & & & $\mathrm{X}$ & $\mathrm{X}$ & & $\mathrm{X}$ \\
\hline $\begin{array}{l}\text { Explicación/ com- } \\
\text { prensión }\end{array}$ & $\mathrm{X}$ & & & & & & & & $\mathrm{X}$ & & $\mathrm{X}$ \\
\hline
\end{tabular}

Tabla 4. Tabulación de las respuestas con respecto a las funciones

Nótese que seis de los profesores sólo reconocen la función verificación/convicción ( $B, D, E, F, G, J)$. Cada uno de los docentes $\mathrm{C}$ y $\mathrm{H}$ le asignan una función más a la demostración: sistematización y descubrimiento, respectivamente. El docente A le otorga dos funciones más a la demostración: sistematización y explicación/comprensión. Los docentes $\mathrm{K}$ e I reconocen todas las funciones de la demostración. Su visión de ésta coincide con lo que el grupo de investigación Aprendizaje y Enseñanza de la Geometría ( $/ Æ \cdot G$ ) de la Universidad Pedagógica Nacional Ilama actividad demostrativa.

\section{Establecimiento de la tipología}

Para finalizar la primera parte del análisis, se procedió a categorizar a los docentes entrevistados, teniendo en cuenta dos grandes aspectos: el primero se refiere a las funciones que le asigna el docente a la demostración (de Villiers, 1993, y Camargo, Samper y Perry, 2006), y el segundo a sus ideas sobre cómo se enseña y cómo se aprende a demostrar (Recio, 2001). Ello porque el análisis anterior muestra que no hay incongruencias significativas entre las ideas respectivas que manifiestan los profesores. Es así que se establecieron tres tipos de visiones.

Tipo A: Corresponde a la visión de los docentes que le asignan a la demostración únicamente la función tradicional de verificación/convicción. Estos docentes consideran que un individuo aprende a demostrar haciendo demostraciones o repitiéndolas. Las demostraciones en el aula de clase hacen parte esencial del contrato didáctico. Sin embargo, no se percibe que tengan interés en realizar actividades que motiven a los estudiantes a producir y entender las demostraciones; es decir, la capacidad para demostrar se convierte en un trabajo personal del estudiante quien imita los ejemplos que el docente muestra. Los docentes que se encuentran clasificados en este tipo son $B, D, E, F, G, J$.

Tipo B: Es la visión de aquellos docentes que le asignan a la demostración todas las funciones. Estos docentes consideran que los estudiantes aprenden a demostrar a través de procesos de exploración, conjeturación y justificación. Además, como de Villiers (1993) lo menciona, al reconocer la 
función de explicación/comprensión ligada a la de descubrimiento, expresan su interés en que la actividad que realiza el estudiante resulte significativa para ellos. Sus visiones de la demostración coinciden con la actividad demostrativa propuesta por el grupo de investigación $\mathbb{E} \cdot G$. También reconocen la demostración como parte esencial del contrato didáctico; realizan acciones para generar el interés en los estudiantes por la demostración. Consideran que la importancia de que los estudiantes realicen demostraciones no está en que las presente de manera rigurosa sino en cómo se construye. Los docentes que se encuentran clasificados en este tipo son $\mathbf{K}$ e $\mathbf{~}$.

Tipo C: Es la visión de los docentes que reconocen la función de verificación/ convicción como la principal de la demostración pero también le asignan una o dos funciones más. Sin embargo, el hecho de que reconozcan otras funciones no lleva a pensar que su idea de demostración coincida con la actividad demostrativa. Algunos de ellos reconocen que se deben generar procesos de exploración y conjeturación para acceder a la justificación, pero no tienen en cuenta que para llevar a cabo esto es necesario promover las funciones de descubrimiento y explicación/comprensión como acciones. Ellos también consideran la demostración como parte esencial del contrato didáctico, mas no se percibe que tengan algún interés en realizar actividades que motiven a los estudiantes a producir y entender las demostraciones, cuestión que los asemeja a los docentes de Tipo A. Los docentes que se encuentran clasificados acá son $\mathbf{A}, \mathbf{C}$, y $\mathbf{H}$.

A continuación se presentan ideas comunes expresadas en la entrevista por los profesores de cada uno de los tipos (Tabla 5).

Tabla 5. Aspectos comunes encontradas en las entrevistas

\begin{tabular}{|c|c|c|c|}
\hline $\begin{array}{l}\text { Número } \\
\text { Pregunta }\end{array}$ & $\begin{array}{c}\text { Ideas características } \\
\text { profesores Tipo A }\end{array}$ & $\begin{array}{c}\text { Ideas características } \\
\text { profesores Tipo B }\end{array}$ & $\begin{array}{c}\text { Ideas características } \\
\text { profesores Tipo C }\end{array}$ \\
\hline 1 & $\begin{array}{l}\text { La demostración es un } \\
\text { proceso para justificar } \\
\text { la veracidad de una } \\
\text { proposición, por medio } \\
\text { de unas reglas lógicas. }\end{array}$ & $\begin{array}{l}\text { La demostración es } \\
\text { un mecanismo con } \\
\text { diferentes funciones. }\end{array}$ & $\begin{array}{l}\text { La demostración se } \\
\text { construye con otros } \\
\text { resultados. }\end{array}$ \\
\hline 2 & $\begin{array}{l}\text { Sí es importante; es la } \\
\text { forma de determinar } \\
\text { la veracidad de las } \\
\text { proposiciones en } \\
\text { matemáticas. }\end{array}$ & $\begin{array}{l}\text { Es el método que } \\
\text { permite validar el } \\
\text { conocimiento. }\end{array}$ & $\begin{array}{l}\text { Sí, ya que permite } \\
\text { convencerse y } \\
\text { determinar la } \\
\text { veracidad de } \\
\text { una proposición } \\
\text { matemática. }\end{array}$ \\
\hline 3 & $\begin{array}{l}\text { Tratar de convencer } \\
\text { de la veracidad de } \\
\text { una proposición a los } \\
\text { estudiantes. }\end{array}$ & $\begin{array}{l}\text { Por medio de la } \\
\text { demostración los } \\
\text { estudiantes realizan } \\
\text { una actividad parecida } \\
\text { a la que realizan los } \\
\text { matemáticos. }\end{array}$ & $\begin{array}{l}\text { Es un medio que } \\
\text { permite desarrollar } \\
\text { pensamiento lógico y } \\
\text { permite comprender } \\
\text { los objetos } \\
\text { matemáticos. }\end{array}$ \\
\hline
\end{tabular}


Aproximación a las visiones de demostración de algunos profesores universitarios de matemáticas Carmen Samper de Caicedo | Yuri Tatiana Ospina Usaquén | Tania Julieth Plazas Merchán

Tabla 5. Aspectos comunes encontradas en las entrevistas (Continuación)

\begin{tabular}{|c|c|c|c|}
\hline 4 & $\begin{array}{l}\text { Se dan algunas pautas } \\
\text { para que el estudiante } \\
\text { aprenda a demostrar, } \\
\text { y se hacen muchas } \\
\text { demostraciones en el } \\
\text { tablero }\end{array}$ & $\begin{array}{l}\text { Diseñando actividades } \\
\text { que sean significativas } \\
\text { para el estudiante } \\
\text { que buscan que el } \\
\text { estudiante realice un } \\
\text { proceso de exploración, } \\
\text { construcción } \\
\text { de conjeturas y } \\
\text { justificación. }\end{array}$ & $\begin{array}{l}\text { Se dan algunas pautas } \\
\text { para que el estudiante } \\
\text { aprenda a demostrar. }\end{array}$ \\
\hline 5 & $\begin{array}{l}\text { Demostrando y } \\
\text { leyendo, entendiendo } \\
\text { y replicando las } \\
\text { demostraciones que } \\
\text { otros han hecho. }\end{array}$ & $\begin{array}{l}\text { Por medio de un } \\
\text { proceso que sea } \\
\text { significativo; este } \\
\text { proceso puede ser } \\
\text { de exploración, } \\
\text { construcción } \\
\text { de conjeturas y } \\
\text { justificación. }\end{array}$ & $\begin{array}{l}\text { Haciendo muchas } \\
\text { demostraciones, } \\
\text { leyendo, entendiendo } \\
\text { y replicando otras } \\
\text { demostraciones, en un } \\
\text { trabajo conjunto con el } \\
\text { profesor. }\end{array}$ \\
\hline 6 & $\begin{array}{l}\text { Para convencerse y } \\
\text { tener certeza de las } \\
\text { afirmaciones que realiza } \\
\text { en clase son verdaderas, } \\
\text { más no para hacerlas } \\
\text { con los estudiantes. }\end{array}$ & $\begin{array}{l}\text { Sí es importante, ya que } \\
\text { la demostración permite } \\
\text { que el profesor tenga } \\
\text { más herramientas para } \\
\text { llevar el conocimiento } \\
\text { al aula de clase y pueda } \\
\text { generar ambientes de } \\
\text { aprendizaje para sus } \\
\text { estudiantes. }\end{array}$ & $\begin{array}{l}\text { Para convencerse y } \\
\text { tener certeza de que } \\
\text { las afirmaciones que se } \\
\text { realizan en clase son } \\
\text { verdaderas; además } \\
\text { para aprender a } \\
\text { argumentar. }\end{array}$ \\
\hline 7 & $\begin{array}{l}\text { Se deben llevar } \\
\text { en un nivel más } \\
\text { elemental, como sólo } \\
\text { argumentaciones. Sin } \\
\text { embargo, muchas de } \\
\text { las demostraciones } \\
\text { dependen del tema } \\
\text { trabajado. }\end{array}$ & $\begin{array}{l}\text { Se deben llevar en un } \\
\text { nivel más elemental, } \\
\text { por medio de un } \\
\text { proceso de exploración, } \\
\text { construcción } \\
\text { de conjeturas y } \\
\text { justificación, el nivel de } \\
\text { justificación debe ser } \\
\text { menos riguroso. }\end{array}$ & $\begin{array}{l}\text { Las demostraciones } \\
\text { en este nivel se deben } \\
\text { llevar de manera } \\
\text { más elemental; se } \\
\text { debe buscar que los } \\
\text { estudiantes aprendan a } \\
\text { argumentar. }\end{array}$ \\
\hline 8 & $\begin{array}{l}\text { Las demostraciones } \\
\text { difieren por los objetos } \\
\text { matemáticos trabajados, } \\
\text { pero el razonamiento } \\
\text { es el mismo. Lo que } \\
\text { se busca con las } \\
\text { demostraciones es } \\
\text { determinar la veracidad } \\
\text { de las proposiciones en } \\
\text { la teoría. }\end{array}$ & $\begin{array}{l}\text { Las demostraciones } \\
\text { difieren por los objetos } \\
\text { matemáticos trabajados, } \\
\text { pero el razonamiento es } \\
\text { el mismo. }\end{array}$ & $\begin{array}{l}\text { Las demostraciones } \\
\text { difieren por los objetos } \\
\text { matemáticos trabajados, } \\
\text { pero el razonamiento es } \\
\text { el mismo. }\end{array}$ \\
\hline
\end{tabular}


- Fase 2 (Análisis de Registro Audiovisual) Los docentes a quienes se les hizo el registro audiovisual fueron $\mathrm{D}, \mathrm{E}$ e I. El objetivo era determinar si había diferencias con las ideas expresadas en la entrevista y ampliar, de ser posible, las ideas que tienen respecto a la demostración.

El docente $D$, durante la entrevista, le asignó como única función a la demostración, la de verificación/convicción. Sin embargo, por medio del análisis del registro audiovisual, se observa que reconoce la función explicación/comprensión, porque el énfasis que hizo, antes de abordar la demostración, fue en cómo se construyen los objetos y las propiedades que se desean demostrar de ellos, a través de varios ejemplos de la situación relacionada con el hecho que se iba a demostrar, cambiando las condiciones del objeto en los ejemplos. Su interés era que los estudiantes visualizaran el teorema.

El docente $E$ permite que sus estudiantes construyan las demostraciones y las expongan ante sus compañeros. Además, en la construcción de la demostración, cuestiona a los estudiantes respecto a las afirmaciones que hacen. Sin embargo, las preguntas que formula a los estudiantes no propician su reflexión respecto a las propiedades del objeto matemático estudiado. El profesor reconoce la función de sistematización; esto porque hace hincapié en la utilización de teoremas demostrados anteriormente, y si alguien sugiere usar algo que no está demostrado, exige su demostración antes de ello.

Lo expresado por el docente I en la entrevista es similar a lo que realiza en el aula, ya que se evidenciaron todas las funciones en la actividad desarrollada en clase. La situación propuesta por el docente para generar la demostración era de carácter explora- torio y de descubrimiento porque solicita que se estudie la relación entre el objeto matemático y una propiedad específica; con ella, el docente busca que los estudiantes comprendan el teorema, antes de tratar de demostrarlo. Además, el profesor hace explícito que toda afirmación se debe validar, exige que las validaciones sean realizadas con recursos disponibles de la teoría, es decir que lo que se utilice ya se haya demostrado, y propicia la participación de los estudiantes en la construcción de la demostración. Para ello, da espacio para que los estudiantes discutan la situación entre ellos y formulen una conjetura que deben justificar. Posteriormente, los estudiantes socializan sus conjeturas y se construye, como comunidad, la demostración.

A pesar de que los docentes $D$ y $E$ reconocen otra función de la demostración, la actividad matemática que realizan en torno a ella en clase no se asemeja a la actividad demostrativa, mientras que lo realizado por el docente I sí está acorde con ella. Sin embargo, se puede afirmar que las visiones de los docentes $D$ y $E$, desde su práctica, no pertenecen al Tipo A sino al Tipo C, ya que su gestión del proceso para demostrar es más rico que lo que expresan de ello. La visión del docente I se mantiene en el Tipo $B$, dado que su práctica y su visión de la demostración coinciden.

\section{Conclusiones}

Como resultado del trabajo, se observa que los docentes de la Licenciatura en Matemáticas de la Universidad Pedagógica Nacional tienen diferentes visiones de la demostración en relación con su función y el propósito que tiene ésta en el aula de clase. Algunas de sus ideas coinciden con lo que en el marco teórico se denominó como la demostración vista desde la educación mate- 
Aproximación a las visiones de demostración de algunos profesores universitarios de matemáticas Carmen Samper de Caicedo | Yuri Tatiana Ospina Usaquén | Tania Julieth Plazas Merchán

mática (Recio, 2001), puesto que reconocen algunas de las funciones de la demostración reportadas por de Villiers (1993). En particular, todos identifican la función de verificación/convicción, y reconocen que la demostración es un recurso necesario en la formación matemática de un individuo.

El análisis de la información obtenida permitió establecer tres tipos de visiones en las que se agrupan ideas similares respecto a la demostración. Tipo A: Se encuentran en este grupo cuatro docentes, quienes reconocen como única función de la demostración la verificación/validación. Además consideran que el aprendizaje de la demostración es un acto individual del estudiante y que el papel del docente es exigir que éste lea, entienda y copie demostraciones. Tipo B: Los dos docentes de esta categoría reconocen todas las funciones de la demostración. Coinciden en que la demostración debe ser una actividad significativa para sus estudiantes. Para ello, generan actividades que propician procesos de exploración, conjeturación y justificación; es decir, coinciden con lo descrito por el grupo de investigación $\mathbb{E} \cdot G$ como actividad demostrativa. Tipo C: Los cinco docentes que fueron clasificados así identifican una o dos funciones más de la demostración aparte de la verificación/convicción. Sin embargo, respecto al aprendizaje asumen que el estudiante debe realizar un trabajo personal para llegar a elaborar una demostración y que aprenden a demostrar replicando demostraciones.

En el proceso realizado, se evidenció que para determinar la visión que tiene un docente respecto a una actividad matemática requiere no solamente de las respuestas proferidas en una entrevista sino también de la observación de la práctica en el aula. En este estudio se caracteriza la visión de que tienen algunos docentes de la demostración, puesto que para determinar la concepción se requiere un estudio más profundo y acopiar mayor información.

Dado el análisis y las conclusiones, surge como inquietud: si los docentes que están formando a los estudiantes de la Licenciatura en Matemáticas de la Universidad Pedagógica Nacional tienen visiones diferentes respecto a la demostración entonces, ¿cuáles son las visiones que tienen estos estudiantes en torno a dicha actividad? y ¿cómo inciden las visiones de los profesores en la formación matemática y didáctica de los futuros educadores matemáticos?

\section{Referencias bibliográficas}

Camargo, I. Samper, C. Perry, P. (2006). Una visión de la actividad demostrativa en geometría plana para la educación matemática con el uso de programas de geometría dinámica. Lecturas Matemáticas. Volumen especial, pp. 371 - 383. Colombia.

De Villiers, M. (1993). El papel y la función de la demostración en Matemáticas. Revista Epsilon, 26, 15-29.

Godino, J. y Recio, A. (2001). Significados Institucionales de la demostración. Implicaciones para la Educación Matemática. Enseñanza de las ciencias. 19 (3), 405 - 414.

Recio, T. (2001). La mecánica de la demostración y la demostración mecánica. En: http://www.uv.es/ didmat/angel/seiem. html , Septiembre de 2008.

Thompson, A. (1992). Creencias y concepciones de los profesores una síntesis de la investigación. D. Grouws (Eds.) Traducido por Moises Ledezma (2001). Manual de investigación sobre enseñanza y aprendizaje de las matemáticas. Capítulo 7, pp. 127143. New York. 